\title{
Aspects juridiques de la révision de la LAMal*
}

Hanspeter Kuhn, avocat, Secrétaire général adjoint de la FMH

\section{Les art. 59 et 59a: un échec total}

\section{Art. 59, al. 3, avant-dernière phrase:} suspension du «droit de pratique»

L'assurance sociale ne peut réglementer que l'admission des fournisseurs de prestations. L'octroi et le retrait du droit de pratique relèvent en revanche uniquement de la compétence des cantons. En d'autres termes, cette disposition est anticonstitutionnelle.

Art. 59a (nouveau): exclusion des seuls fournisseurs de prestations médicales («ärztliche Leistungserbringer»)

Ainsi donc, selon la loi, les chiropraticiens, les sages-femmes et les «institutions de soins ambulatoires dispensés par des médecins» au sens de l'art. 36a, LAMal, ne sauraient en aucun cas travailler de manière trop dispendieuse.

\section{Al. 1: limite de $10 \%$ pour les procédures de remboursement}

Cette limite s'applique-t-elle à la moyenne globale des prestations fournies, ou suffit-il de la dépasser dans un seul paramètre pour déclencher une procédure?

Mais encore: il apparaît que la gravité de la maladie ne joue manifestement aucun rôle en ce qui concerne l'avertissement à donner. Ceci contredit l'état actuel des connaissances en matière d'évaluation du profil des médecins (Physician profiling): il est en effet internationalement admis que cet aspect doit être pris en compte («The issue of severity must be addressed»), car les médecins ne traitent pas tous le même nombre de patients qui coûtent cher [1].

1 Cf., parmi beaucoup d'autres, Kongstvedt PR. Essentials of Managed Health Care. $4^{\text {th }}$ edition. Gaithersburg, MD: Aspen Publication; 2001 ISBN 0-8342-1862-3, p. 401.

2 "The problem of provider specialty definition is particularly acute when looking at primary care. [...] Many board-certified medical specialists spend a considerable amount of time performing primary care, whereas others spend the majority of their time practicing true specialty medicine. [...] Even within a single specialty there will be difference in how 'specialized' a specialist is." [1], p. 398.
Fin de l'al. 3: suppression du numéro d'identification en tant que sanction Le numéro d'identification est une prestation de service privée, et juridiquement peu importante, de santésuisse (ce que le Conseil fédéral a reconnu à juste titre lorsqu'il a édicté l'ordonnance du 3 juillet 2002 concernant la clause du besoin).

\section{Al. 4: groupe de référence composé exclusivement selon le critère du titre de formation postgraduée}

Ceci est également en contradiction avec le savoir en la matière disponible au niveau interna- tional: «The specialty of the physician is not always clear.» [2]. C'est de manière très inégale que les spécialistes pratiquent aussi en qualité de médecins de famille. Quant à ces derniers, ils ne centrent pas tous leur activité en cabinet sur les mêmes priorités (intégrant, par ex., plus ou moins de psychiatrie à la médecine de premier recours). Le groupe de référence ne saurait donc être composé uniquement en fonction du titre de spécialiste.

\section{Al. 5: une statistique reconnue par tous sert} de base, mais en cas de désaccord, c'est celle des assureurs qui est déterminante

Ce passage se passe de tout commentaire.

\section{Al. 6: les dépens sont mis à la charge} du fournisseur de prestations, à raison de sa culpabilité

Apparemment, le législateur ne peut imaginer qu'il pourrait aussi arriver aux caisses d'être «coupables», donc de ne pas obtenir gain de cause (et de devoir alors supporter les coûts de la procédure).

\section{Al. 7: les recours contre les décisions de la commission paritaire n'ont pas d'effet suspensif}

Le TFA constatera peut-être au bout de deux ans que tel médecin n'aurait pas dû être exclu. Son cabinet étant resté fermé pendant toute cette période, qui paiera le dommage occasionné?

\section{Dispositions discutables}

\section{Art. 2: suppression du verbe «qui exige»}

On fait ici table rase d'un critère juridique ayant fait ses preuves pendant des décennies, selon lequel la caisse ne doit payer que lorsque les troubles traités ont valeur de maladie.

\section{Art. 35: contrat en tant que critère légal d'admission}

La LAMal actuelle opère une distinction entre les critères d'admission tels que la formation et la formation postgraduée d'une part, et la réglementation de la collaboration entre parties d'autre part (convention ou tarif de remplacement fixé par l'autorité compétente). Et voici que 
l'existence même d'une convention devrait devenir un critère d'admission supplémentaire. Ceci représente un manquement grave tant du point de vue politique que législatif. En effet, les caisses deviendraient ainsi des organisations chargées d'octroyer des concessions (c'est-à-dire des admissions par le biais de conventions): une réglementation inacceptable, car, comme l'indiquent clairement ne fût-ce que les articles $85 \mathrm{ss}$ LAMal consacrés aux voies de droit, les partenaires tarifaires sont placés sur le même pied: la caisse ne peut rendre de décision vis-à-vis du médecin comme elle peut et doit le faire à l'égard du patient.

A supposer qu'elle fût nécessaire, une telle disposition aurait plutôt sa place dans le contexte de l'article 25 ou de l'article 32 . Nous souhaitons au demeurant un plein succès au Conseil fédéral au cas où il devrait définir (selon le vœu de la minorité de la commission), quels sont les cardiologues qui exercent en qualité de généralistes et quels sont ceux qui pratiquent en tant que spécialistes...

Art. 39 f: médecine de pointe, ainsi que I'art. 39a, nouveau (minorité): équipements techniques lourds

Ici également, on peut n'être que curieux des délimitations qu'il s'agirait d'opérer: qu'est-ce que la médecine de pointe? Comment définir les équipements techniques lourds («Grossgeräte» au mètre cube)?

\title{
Loi sur I'assurance-maladie. Révision partielle (financement des hôpitaux)
}

\author{
Conseil national, session d'hiver 2002, 00.079 é
}

Extraits du projet de révision de la LAMal actuellement débattu par le Conseil national. Seules sont reproduites les dispositions commentées par Hanspeter Kuhn dans son article ci-avant. L'ensemble du projet, tout en n'étant pas tenu secret, ne peut être consulté sous forme électronique.

Art. 59, al. 3

Proposition de la Commission de la sécurité sociale et de la santé publique (CSSS) du Conseil national (nouveau)

3 Les fournisseurs de prestations qui ne se conforment pas aux exigences de qualité et d'économicité du traitement prévues par les articles 56 et 58 ainsi que les conventions avec les assureurs en vigueur font l'objet de sanctions. Celles-ci comportent la restitution de tout ou partie des honoraires touchés pour des prestations fournies de manière inappropriée, l'amende, et la suspension temporaire ou définitive du droit de pratique [termes mis en italique par la $\mathrm{FMH}$ ] en cas de récidive. Le tribunal arbitral au sens de l'article 89 en décide.

Art. 59a

Minorité de la CSSS du Conseil national: avertissement, et exclusion de fournisseurs de prestations médicales pour mauvaise gestion du cabinet (nouveau)

$1 \mathrm{Si}$, pour un fournisseur de prestations médicales, les dépenses par patient et par an pour des prestations qu'il a fournies lui-même ainsi que pour des médicaments, des analyses de laboratoire et des radiographies dépassent de plus de $10 \%$ la moyenne arithmétique de son groupe de référence, il lui est donné un avertissement par une commission cantonale composée paritairement de fournisseurs de prestations et d'assureurs.

2 Le fournisseur de prestations médicales dispose de trois mois à compter de la réception de l'avertissement pour adresser par écrit à la commission un recours par lequel il apporte la preuve que le dépassement concerné est dû à la structure spécifique et la composition des patients ou à d'autres circonstances extraordinaires et que la gestion de son cabinet ne contrevient en rien aux principes d'une gestion saine. 
3 A la fin de la deuxième année suivant l'avertissement, la commission décide ou non d'interdire aux fournisseurs de prestations pour une ou plusieurs années, et définitivement en cas de récidive, de remplir les missions visées par la présente loi, si l'écart au sens de l'al. 1 est toujours supérieur à $10 \%$ et si la commission a estimé que la preuve visée à l'al. 2 n'a pas été apportée. La décision est communiquée au service compétent des assureurs afin qu'il procède à la suppression du numéro d'identification.

4 Le groupe de référence est composé des fournisseurs de prestations titulaires des mêmes diplômes fédéraux de formation continue actifs dans le canton ou, s'il compte moins de 15 membres, au niveau de la Confédération.

5 L'avertissement et la procédure d'exclusion sont fondés sur une statistique reconnue par les fournisseurs de prestations et par les assureurs. A défaut de statistique reconnue par tous, la statistique centrale des assureurs est réputée déterminante.

6 Nommée par le gouvernement cantonal, la commission paritaire est composée de deux représentants des associations cantonales des fournisseurs de prestations et de deux représentants des assureurs. Le gouvernement nomme un président neutre, qui tranche en cas d'égalité des voix. La commission tranche dans un délai de six mois au moyen d'une décision écrite brièvement motivée. Elle peut demander des informations complémentaires auprès du fournisseur de prestations concerné et des assureurs, et s'adjoindre le concours d'experts. Par ailleurs, elle décide elle-même de la procédure. Les dépens sont mis à la charge du fournisseur de prestations, à raison de sa culpabilité. La commission peut fixer un tarif unique.

7 Les décisions prises par la commission peuvent faire l'objet d'un recours auprès du tribunal arbitral cantonal visé à l'art. 89. Le recours n'a pas d'effet suspensif.

Art. 2 Définitions

Art. 35 Principe

\section{Droit en vigueur}

1 Par maladie, on entend toute atteinte à la santé physique ou mentale qui n'est pas due à un accident et qui exige un examen ou un traitement médical ou provoque une incapacité de travail.

\section{Proposition de la CSSS du Conseil national (nouveau)}

1 Par maladie, on entend toute atteinte qui

$[\ldots]$

[...] provoque un examen ou un traitement médical ou des soins.

\section{Droit en vigueur}

1 Sont admis à pratiquer à la charge de l'assurance obligatoire des soins les fournisseurs de prestations qui remplissent les conditions des articles 36 à 40 .

\section{Conseil des Etats}

1 Sont admis à pratiquer à la charge de l'assurance obligatoire des soins les fournisseurs de prestations qui:

a. remplissent les conditions des articles 36 à 40, et

b. pour autant qu'ils soient actifs dans le secteur ambulatoire, ont conclu un contrat d'admission avec un ou plusieurs assureurs-maladie reconnus.

\section{Minorité de la CSSS du Conseil national}

1 Sont admis à pratiquer à la charge de l'assurance obligatoire des soins les fournisseurs de prestations qui:

a. remplissent les conditions des articles 36 à 40;

b. exercent en qualité de généraliste dans le secteur ambulatoire;

c. exercent en qualité de spécialiste dans le secteur ambulatoire et ont conclu un contrat d'admission avec un ou plusieurs assureurs; 
Art. 39 Hôpitaux et autres institutions d. sont affiliés à un modèle de médecin de famille agréé et se conforment à la discipline budgétaire définie à l'article 35 a (nouveau).

Le Conseil fédéral peut décider, par voie d'ordonnance, d'inclure dans le champ d'application de la let. b des prestations de médecine spécialisée relevant du domaine de la couverture générale.

$[\ldots]$.

\section{Droit en vigueur}

1 Les établissements et celles de leurs divisions qui servent au traitement hospitalier de maladies aiguës ou à l'exécution, en milieu hospitalier, de mesures médicales de réadaptation (hôpitaux) sont admis s'ils:

[...]

e. figurent sur la liste cantonale fixant les catégories d'hôpitaux en fonction de leurs mandats.

\section{Majorité de la CSSS du Conseil national (nouveau)}

f. dans le domaine de la médecine de pointe, correspondent à la planification établie par la Confédération.

\section{Minorité de la CSSS du Conseil national (nouveau)}

a. Pour assumer la maîtrise des coûts de la santé et pour sauvegarder un intérêt public prépondérant, la mise en service d'équipements techniques lourds ou d'autres équipements de médecine de pointe, dans le domaine hospitalier ou ambulatoire, public ou privé, est soumise à l'autorisation du canton concerné. 